Bull. Austral. Math. Soc.

VOL. $72(2005)$ [335-336]

\title{
Geometric Seifert 4-manifolds with hyperbolic bases
}

\author{
MiCHAEL KEMP
}

Seifert fibred 3-manifolds were originally defined and classified by Seifert in [2]. Scott (in [1]) gives a survey of results connected with these classical Seifert spaces, in particular he shows they correspond to 3-manifolds having one of six of the eight 3-dimensional geometries (in the sense of Thurston). Essentially, a classical Seifert manifold is a $S^{1}$. bundle over a 2-orbifold. More generally, a Seifert manifold is the total space of a bundle over a 2-orbifold with flat fibres. It is natural to ask if these generalised Seifert manifolds describe geometries of higher dimension. Ue has considered the geometries of orientable Seifert 4-manifolds (which have general fibre a torus) $([3,4])$. He proves that (with a finite number of exceptions) orientable manifolds of eight of the 4-dimensional geometries are Seifert fibred. However, Seifert manifolds with a hyperbolic base are not necessarily geometric. In this paper, we seek to extend Ue's work to the non-orientable case.

Firstly, we shall show that Seifert spaces over an aspherical base are determined (up to fibre preserving homeomorphism) by their fundamental group sequence. Furthermore when the base is hyperbolic, a Seifert space is determined (up to fibre preserving homeomorphism) by its fundamental group. This generalises the work of Zieschang ([5]), who assumed the base has no reflector curves, the fibre was a torus and that a monodromy of a loop surrounding a cone point is trivial. Then we restrict to the 4 dimensional case and find necessary and sufficient conditions for Seifert 4 manifolds over hyperbolic or Euclidean orbifolds to be geometric in the sense of Thurston. Ue proved that orientable Seifert 4-manifolds with hyperbolic base are geometric if and only if the monodromies are periodic, and we shall prove that we can drop the orientable condition. Ue also proved that orientable Seifert 4-manifolds with a Euclidean base are always geometric, and we shall again show the orientable assumption is unnecessary.

\section{REFERENCES}

[1] P. Scott, 'The geometries of 3-manifolds', Bull. London Math. Soc. 15 (1983), 401-487.

[2] H. Seifert, 'Topologie dreidimensionaler gefaserter Räume', Acta Math. 60 (1933), 147-238.

Received 20th August, 2005

Thesis submitted to The University of Sydney, September 2004. Degree approved, August 2005. Supervisor: Dr. Jonathan Hillman.

Copyright Clearance Centre, Inc. Serial-fee code: 0004-9727/05 \$A2.00+0.00. 
[3] M. Ue, 'Geometric 4-manifolds in the sense of Thurston and Seifert 4-manifolds I', $J$. Math. Soc. Japan 42 (1990), 511-540.

[4] M. Ue, 'Geometric 4-manifolds in the sense of Thurston and Seifert 4-manifolds II', $J$. Math. Soc. Japan 43 (1991), 149-183.

[5] H. Zieschang, 'On toric fibreings over surfaces', Math. Zametki. 5 (1969), 569-576. English translation Math. Notes. 5, (1967), 341-345.

17 Sheldon Crescent

Orange NSW 2800

Australia

e-mail: mkemp@csu.edu.au 